\title{
L'enchevêtrement des temporalités : eschatologie et corruption dans Le Decez, ou fin du monde (1584) de Guillaume de Chevalier
}

\section{Adrien Paschoud}

\section{(2) OpenEdition \\ Journals}

Édition électronique

URL : http://journals.openedition.org/edl/903

DOI : $10.4000 /$ edl. 903

ISSN : 2296-5084

Éditeur

Université de Lausanne

\section{Édition imprimée}

Date de publication : 15 décembre 2015

ISBN : 978-2-940331-47-5

ISSN : 0014-2026

Référence électronique

Adrien Paschoud, «L'enchevêtrement des temporalités : eschatologie et corruption dans Le Decez, ou fin du monde (1584) de Guillaume de Chevalier », Études de lettres [En ligne], 3-4 | 2015, mis en ligne le 01 décembre 2018, consulté le 18 décembre 2020. URL : http://journals.openedition.org/edl/903 ;

DOI : https://doi.org/10.4000/edl.903 


\section{L'ENCHEVÊTREMENT DES TEMPORALITÉS: ESCHATOLOGIE ET CORRUPTION DANS LE DECEZ, OU FIN DU MONDE (1584) DE GUILLAUME DE CHEVALIER}

Cet article se propose d'aborder l'expression de la corruption dans Le Decez, ou fin $d u$ monde (1584) de Guillaume de Chevalier, un poème épique d'inspiration biblique. L'auteur élabore une paraphrase de l'Apocalypse et conjugue, dans le prolongement de saint Augustin, deux temporalités, l'une terrestre, l'autre céleste, afin de rendre compte des «maux présents" qui accaparent les hommes avant le Jugement. Usant du motif traditionnel du songe - qui est en réalité une vision -, le poète entend alors conduire l'univers à son néant, peignant ainsi la cohorte de maux - guerre, famine, peste - qui s'abattront successivement sur une humanité corrompue. Si le temps de la maladie est un temps de préparation à la mort, il est le préalable à la renovatio spirituelle et, par extension, politique. En effet, les maux présents, aussi insupportables qu'ils soient, précèdent l'avènement d'une justice véritable, celle qui bannira les vaines hiérarchies mondaines, faisant ainsi taire les divergences confessionnelles qui déchirent le royaume de France.

Composé de quatrains d'alexandrins en rimes croisées, Le Decez, ou fin du monde, divisé en trois visions (1584) de Guillaume de Chevalier ${ }^{1}$ relève d'un genre littéraire dont la diffusion fut grande au tournant des XVI ${ }^{\mathrm{e}}$ et XVII ${ }^{\mathrm{e}}$ siècles: l'épopée d'inspiration apocalyptique ${ }^{2}$. Les œuvres que l'on regroupe sous cette appellation - dont Les Tragiques (1616) d'Agrippa d'Aubigné forment sans nul doute l'acmé - sont indissociablement liées à la crise sans précédent qui a ébranlé le royaume des derniers Valois, avant l'instauration sous le règne d'Henri IV d'une

I. G. de Chevalier, Le Decez, ou fin du Monde, divisé en trois visions.

2. Voir B. Méniel, Renaissance de l'épopée, p. 290-300. 
paix relative, marquée cependant par la hantise du complot politique. La violence inouïe qui s'est emparée aussi bien du clan catholique que protestant dès 1562 a cristallisé à n'en pas douter un puissant imaginaire eschatologique, accentué en outre par les nombreux "prodiges" célestes observés entre 1577 et $1585^{3}$. Quelle que soit leur obédience confessionnelle, les poètes entendent traduire les «signes» - ces «lettres hiéroglyphiques enfantées par la profonde obscurité » - qui président à l'anéantissement par le feu d'un "misérable siècle " ${ }^{4}$ avant le règne éternel de Dieu. Reposant essentiellement, mais non exclusivement, sur la réécriture du récit johannique ${ }^{5}$, les épopées bibliques offrent alors une réponse théologico-littéraire aux désastres présents qu'elles réfractent différentiellement. Elles se nourrissent en amont de l'interprétation augustinienne, largement admise dans la culture médiévale et renaissante, d'après laquelle l'Apocalypse appelle un double régime causal, à la fois historique et métaphysique ${ }^{6}$. Marqué d'une forte empreinte néo-

3. Sur les résonances littéraires de ces phénomènes (apparition probable d'une nova, passage d'une comète en 1577), voir I. Pantin, La poésie du ciel en France dans la seconde moitié du seizième siècle, p. 457-494.

4. G. de Chevalier, Le Decez, ou Fin du Monde, «Epître», p. iii et iv. Opérant une jonction entre sens littéral et sens figuré, le terme de "hiéroglyphe» a toute son importance ici: le Dieu caché ne peut être approché dans sa vérité que par un alphabet mystérieux dont le déchiffrement accroît le désir spirituel et affine l'âme; le hiéroglyphe participe de la fascination de la culture des $\mathrm{XVI}^{\mathrm{e}}$ et $\mathrm{XVII}{ }^{\mathrm{e}}$ siècles pour l'emblématique (voir A.-E. Spica, Symbolique humaniste et emblématique). Ces signes font autant appel aux facultés imaginatives qu'intellectuelles du lecteur, car ils présentent la caractéristique de révéler et d'obscurcir conjointement les mystères sacrés, disposant ainsi puissamment l'âme à la méditation.

5. Aux côtés de l'Apocalypse sont convoqués les livres de Daniel (en particulier le songe de Nabuchodonosor II, 31-33) et d'Ezéchiel, ainsi que l'Evangile de Matthieu (XXIV-XXV) et la Seconde Epître aux Thessaloniciens (II, 1-12).

6. La conjonction des deux temporalités est issue de la pensée augustinienne sous l'égide de laquelle s'ouvre Le Decez, ou fin du monde (l'évêque d'Hippone est présenté comme «un pilote», "avec lequel [le poète a] pensé ne pouvoir faire naufrage parmi ces écueils et détroits de la raison humaine", "Aux lecteurs", p. vii). Dans La Cité de Dieu s'opère une synthèse décisive s'agissant du récit johannique, dont l'influence sera considérable. Le récit de l'Apocalypse n'exclut en aucune manière des résonances aux événements historiques, en dépit de son caractère a priori anhistorique. Saint Augustin dénonce toutefois l'application caricaturale et excessive d'une lecture strictement allégorique et s'en prend aux faux prophètes (d'après Matthieu XXIV, 24), ce que Guillaume de Chevalier dénonce également dans son "Epître", lorsqu'il s'agit de combattre les "fols présages qui échappent à quelques frénétiques» (p. iv). Prédire la date exacte de l'Apocalypse, c'est 
platonicienne, Le Decez, ou fin du monde de Guillaume de Chevalier subordonne la temporalité mondaine à l'atemporalité divine, trouvant dans la figuration de la corruption, ainsi que nous le verrons, un point d'articulation entre la nature et la grâce.

"Ayant pris le vol d'un peu plus haut, je me suis efforcé de représenter au vif dans ce petit œuvre l'état des choses suivant les dernières écritures saintes", lit-on dans les pages liminaires du Decez, ou fin du monde («Epître», p. ii). Se faisant contemplateur des vérités sacrées, Guillaume de Chevalier ambitionne de rendre ce que le récit de l'Apocalypse a voilé d'obscurité; guidé par l'Esprit, le poète a la charge et l'insigne honneur de poursuivre la voix de l'évangéliste Jean. Si toute révélation est achevée depuis l'avènement du Christ, cela n'exclut toutefois pas que le poète ne puisse mimer la parole prophétique, bien au contraire. La poésie de dévotion trouve ici sa pleine légitimité au regard de la théologie, dont elle constitue la précieuse alliée. C'est donc une profération, davantage qu'une narration des fins dernières (Guillaume de Chevalier ne suit que très lâchement la succession des visions johanniques ${ }^{7}$ ), qui est livrée ici, faisant du récitant un véritable réceptacle des mystères divins. La poésie épique consacre dès lors l'élection d'un individu qui livre en retour un témoignage à valeur universelle. Fondé sur un regard surplombant au travers duquel s'affirme l'étroite cohésion du signe et du référent ${ }^{8}$, l'ouvrage de Chevalier ambitionne donc de "déplier»

se livrer à la divination, vivement condamnée dans La Cité de Dieu. La récusation augustinienne des interprétations millénaristes (d'après Apocalypse XX, 1-6) se fonde sur l'idée selon laquelle les mille ans peuvent représenter «toutes les années de ce siècle», en tant "qu'image de cette totalité» temporelle, ou les mille dernières années avant le repos éternel, c'est-à-dire depuis l'incarnation jusqu'au jugement. Voir Th. Victoria, Un livre de feu dans un siècle de fer, p. 84-116.

7. La dispositio du poème témoigne de la relative souplesse du matériau épique dans le traitement de la matière johannique: Guillaume de Chevalier ne consacre aux fléaux annoncés dans l'Apocalypse qu'une portion des plus congrues; s'il est fait allusion à l'imminence de la destruction du monde dans les livres I et III, ces derniers évoquent d'abord et avant tout les grandeurs célestes; quant au livre II, il est voué presque exclusivement à la glorification du sublime divin. De ce point de vue, Le Decez, ou fin $d u$ monde se situe aux frontières du genre épique (si on le compare à l'œuvre de Michel Quillian, pleinement centrée sur la réécriture de l'Apocalypse).

8. L'œuvre de Chevalier voue une confiance absolue au pouvoir mimétique du discours poétique: la présence massive de l'hypotypose invalide d'emblée tout écart qui pourrait se glisser entre les res et les verba. Si l'idée selon laquelle le langage s'est altéré de manière irréversible depuis la Chute est largement répandue à la Renaissance (voir 
l'âpreté et la brièveté du texte johannique afin d'avertir de la nécessité d'agir vertueusement. L'entreprise ne saurait cependant se confondre avec un dépeçage herméneutique ou exégétique, car le poète admet que les mystères divins ne peuvent jamais être pleinement saisis ${ }^{9}$. En lieu et place de cela, Le Decez, ou fin du monde puise dans l'Apocalypse pour construire un artefact littéraire des vérités les plus élevées qui se mue en un opérateur de croyance. A la lecture des vers, l'âme doit s'élever du sensible au spirituel, de la multiplicité des images à l'unité du sens; la méditation spirituelle est alors générée par une alliance des facultés de l'âme. Il s'agit dans le même temps de maintenir une hiérarchie stricte entre poésie de dévotion et texte sacré. Tenu de respecter le dogme, le poète aura alors tout pouvoir de parfaire la représentation des choses dernières.

"Représenter au vif» les fins dernières (on aura reconnu le principe de l'enargeia), c'est pourtant se heurter d'emblée à une double difficulté, inhérente à la prédiction et à la prédication johanniques. L'Apocalypse, au sens étymologique de "révélation", induit un décentrement à la fois spatial et temporel. Spatial, car il s'agit de rendre stylistiquement l'inscription des êtres et des choses dans un ordre divin étranger à toute raison, et dont le principe ne repose bien évidemment pas sur une contigüité pacifique des éléments; temporel, car la matière poétique se donne pour tâche de dévoiler des événements à venir dont la mystérieuse densité échappe au sens commun. Dès lors, toute tentative d'enserrer l'espace et le temps eschatologiques dans une configuration stable de représentation se heurte à une aporie dans la mesure où la mise en discours des fins dernières invalide le principe d'éternité propre à l'ordre divin. On sait que saint Augustin avait fait état de cet obstacle dans le livre XI des Confessions: le langage qui est donné pour saisir le verbe créateur et éternel de Dieu est lui-même temporel; aussi ne peut-on saisir le temps autrement que dans le moment de l'écriture car celui-ci possède

M.-L. Demonet, La voix du signe), elle n'invalide pourtant pas la conjonction entre le langage et l'idéal d'une transparence au second degré. La conception néo-platonisante qui conditionne la poésie dans ses registres les plus élevés fait du langage un outil mutilé, ayant perdu sa transparence originelle; dans le même temps, pourtant, la langue poétique en dépit de ses imperfections, peut approcher l'idée de Dieu; elle n'est donc aucunement vaine. 
la propriété de n'apparaître qu'en disparaissant ${ }^{10}$. Pour contourner ce qui apparaît à bien des égards comme une impossibilité pure et simple, Guillaume de Chevalier, à l'image de ses contemporains (dont Michel Quillian et, plus tard, Agrippa d'Aubigné dans Les Tragiques) a recours au motif traditionnel du songe ${ }^{11}$. Il s'agit plus précisément de trois "visions", "trois beaux mondes" qu'une "beauté suprême», sous la forme d'une lumière étincelante, présente au poète-narrateur; investi du furor d'inspiration néo-platonicienne, ce dernier devra alors s'atteler à les rendre fidèlement. Le songe est l'extrapolation mimétique de la vision johannique, la parole poétique se trouvant cautionnée par les premières lignes de l'Apocalypse: "Ce que tu vois, écris-le» (I, 11). Distinct du «rêve» et de la "rêverie» ${ }^{12}$ car soumis à la volonté et à la raison du poète, le songe fait dialoguer les niveaux interprétatifs, entre l'ici-bas et l'audelà, entre perte et espérance, trouvant un mode d'intellection de ce qui est par essence ineffable. Le poème joue de cet écart entre le visionnaire - Guillaume de Chevalier livre une "vision» de l'univers dans son présent et dans son devenir - et le prophétique, entre le temps de l'énonciation - le hic et nunc des maux qui accablent l'humanité - et le projet divin rétif à toute forme d'explicitation poétique. Il y a là une expérience de pensée, ou mieux une fiction théorique, que le poète se plaît à monter et à démonter, et qui n'est pas sans faire songer à l'évocation du chaos à laquelle se livrera plus tard Descartes dans le livre cinquième du Discours de la méthode.

Initié en songe, le poète, véritable messager de Dieu, touche à une vérité cachée; il prend alors en charge l'imaginaire eschatologique et donne à voir des entités étrangères au sens commun. Faisant état de l'origine divine de son âme (on reconnaît ici l'anamnèse platonicienne

9. Cela n'a rien d'original. Guillaume de Chevalier est fidèle à l'idée selon laquelle le langage poétique ne contient pas tout ce qu'il exprime: la poésie de dévotion construit certes une totalité, mais n'est pas à même d'en épuiser tous les ressorts.

Io. Voir en particulier les chapitres XV et XVI du livre XI des Confessions.

II. Voir les études réunies dans Le Songe à la Renaissance, éd. par F. Charpentier, notamment l'introduction de S. Perrier, "La problématique du songe à la Renaissance», p. 13-19.

I2. A l'exception de Montaigne, la "rêverie» a généralement une connotation négative au XVI ${ }^{\mathrm{e}}$ siècle, celle de l'abandon aux sens trompeurs; elle peut également désigner un raisonnement aberrant (Calvin a souvent recours à ce terme pour stigmatiser ses ennemis, cf. Contre les libertins [1544], in Euvres, p. 797, p. 807 et p. 809). Sur l'étymologie du mot "rêverie», voir F. Orwat, L'Invention de la rêverie. 
comme souvenir d'un état idéal de l'âme antérieur à son incarnation), le poète est alors doué du pouvoir de faire converger dans un même espace de représentation le passé, le présent et l'avenir - en ce sens, le songe dépasse de loin la fonction de fable vraisemblable que lui attribuaient les commentateurs de Macrobe, via le De Divinatione de Cicéron, dans la mesure où le récit de "vision» est destiné à porter les vérités les plus élevées ${ }^{13}$. Pour cela, le domaine poétique doit se laisser envahir par l'éloquence sacrée. Tissant un lien entre le visible et l'invisible, entre le littéral et le figuré, cette dernière fait montre des qualités descriptives du poète: il lui incombe en effet d'élaborer des "tableaux" ("Aux lecteurs», p. vii) prompts au surgissement, via le procédé de l'hypotypose, des mystères sacrés. Conformément à la doctrine de l'ut pictura poesis, la "peinture" des fins dernières oscille entre description et invocation; mêlant lucidité et évidence lumineuse dans le prolongement du sublime chrétien ${ }^{14}$, elle se donne pour tâche de ravir l'esprit du lecteur, sans toutefois former un substitut du texte biblique, entreprise bien évidemment impensable. Car céder aux seules chimères de l'imagination et aux vains plaisirs de l'ingenium serait bien entendu hautement condamnable; le lecteur serait alors abandonné aux reflets trompeurs des signes; fasciné par les beautés de l'ornatus, il substituerait au divin les représentations du divin, versant en cela dans l'idolâtrie (c'est là l'héritage des débats relatifs aux représentations du divin dans le sillage des écrits de Denys l'Aréopagite ${ }^{15}$ ). Parce qu'il est soumis à la recréation de la parole divine, le motif du songe est soustrait à tout soupçon de fausseté ou de délire imaginatif ${ }^{16}$.

I3. Le Songe de Scipion, inséré dans La République de Cicéron, a connu une postérité considérable au XVI ${ }^{\mathrm{e}}$ siècle. Voir S. Lecompte, La chaîne d'or des poètes, p. 207-247. Le poème de Chevalier emprunte au Songe de Scipion le topos du voyage céleste (p. 3).

I4. Le Traité du sublime attribué à Longin paraît en 1554, avant de connaître de nombreuses rééditions. On rappellera que le traité de Longin réconcilie l'art et le génie, la mania platonicienne et la technè d'Aristote: le sublime ne saurait se situer exclusivement du côté de la furor; elle implique une approche analytique seule à même de restituer l'ineffable.

15. Voir notamment la Hiérarchie céleste, II, 3 (140C et 141A) dans laquelle Denys l'Aréopagite traite de l'usage des symboles dans la représentation du divin, les jugeant dangereux. Voir de manière plus générale, J. Miernowski, Signes dissimilaires.

I6. Il s'agit pourtant d'une utopie tant les paraphrases poétiques des textes sacrés sont saturées de figures de style. Le Decez, ou fin du monde affiche un goût prononcé pour les rapprochements surprenants et les montages ingénieux, touchant par moments à une forme d'abstraction qui dépasse de loin la tension interprétative présupposée par le sens 
$\mathrm{Au}$ vu de ce qui précède, le recours au songe, artifice commode qui légitime la démarche spirituelle du poète, en même temps que le projet d'écriture lui-même, permet de dérouler dans la temporalité linéaire de l'écriture une temporalité qui lui est étrangère, car toujours différée au vu de sa composante prophétique: n'est-ce pas une "horloge sans heures» (p. 4) qui guide le devenir de l'humanité? Surtout, par la perspective surplombante qu'il offre, défiant toute contingence, le songe est la clé poétique qui unit la grande narration biblique du commencement et de la fin, défaisant à rebours les grandeurs de la Création: "Le sujet est grand et semble désirer un plus long volume vu qu'à la dissolution du monde n'y a pas moins de matière pour discourir qu'à sa création " peut-on lire dans l'«Epître» (p. ii). L'avenir funeste d'un univers voué à s'altérer inéluctablement est contenu en germe dans le moment fondateur de la Genèse (la "Genèse" est au sens premier une "naissance", mais aussi, dans une signification seconde, un "destin "); l'Apocalypse marque théologiquement la fin de l'histoire dont elle dévoile a posteriori la signification, conformément à son étymologie hébraïque ${ }^{17}$. Dès lors, la conjonction de la temporalité humaine et de l'atemporalité divine ne conduit aucunement à l'évocation d'un monde en proie à l'indistinction; elle asseoit bien au contraire un processus qui tend vers l'ordre renouvelé de Dieu, suivant en cela la Seconde Epître aux Thessaloniciens (II, 1-12):

[...] lorsque toutes les choses lesquelles naissantes et mourantes par les révolutions assiduelles des cercles parfont le monde seront revenues à leur premier point, [l'homme pourra] être de ceux qui prendront la couronne triomphante d'immortalité» («Epître», p. iii).

En ce sens, la rupture de la concordia discors n'a rien d'un chaos élémentaire, celui qui caractérisait par exemple la Théogonie d'Hésiode; elle n'a rien non plus d'une conception de la nature, considérée comme le lieu de forces aveuglément brutales, qui serait livrée à elle-même, chose impensable dans une optique chrétienne; si la matière semble être la

spirituel. Sous le règne d'Henri IV, nombreux seront ceux qui préconiseront une théorie de la mesure - et plus spécifiquement un usage restreint de la métaphore - en matière d'écriture spirituelle.

I7. Galeh («révélation») en hébreu (le terme se trouve en Daniel II, 19;22;28; Amos III, 7; Proverbes XI, 13; Matthieu XI, 27). 
proie de tous les désordres, elle n'en demeure pas moins soumise à la volonté divine.

On ne s'étonnera, dès lors, que la conception du temps énoncée dans Le Decez, ou fin du monde soit fondée sur ce que nous pourrions nommer un mouvement de réfraction et d'expansion; le retour à la destruction et au chaos est un mouvement inhérent au dessein divin, avant de conduire in fine à une harmonie parfaite:

Le monde corruptible est de manière instable,

Principe de sa mort, il a commencement:

Toute chose qu'on voit dans ce clos habitable

Avec l'esprit vital, prend fin du mouvement (p. 3).

La fragmentation des quatre éléments relève d'un principe universel d'ordre métaphysique qui reflète, en retour, l'ordonnancement originel de celui-ci; elle relève de la récapitulation, d'origine paulinienne (Ephésiens, I, 10), qui veut que l'ensemble des choses créées par le Verbe convergent in fine vers un point unique, une sublimation ou une transfiguration des êtres sous la bannière du Christ ${ }^{18}$. La vision du poète est cautionnée bibliquement par les bouleversements cosmiques (obscurcissement du soleil, chute des étoiles, ébranlement des astres et des forces célestes) qui précèdent la parousie selon Matthieu, XXIV, 29-31. La restitution du règne de Dieu ne peut qu'émaner de la dégradation du temps profane. Il y a là un paradoxe que le poète, s'appuyant sur la pensée platonicienne ${ }^{19}$, peut aisément résoudre: le désordre de la matière fait partie en tous points de l'intelligible et touche alors à une pensée de l'origine; selon les cosmologies renaissantes de stricte obédience, Dieu a créé la matière avant toutes choses (la matière ne saurait préexister au logos sous peine de verser dans la tradition lucrétienne). Bien qu'elle puisse subir de violentes altérations, la matière est toujours en quantité égale, elle est coextensive à l'action de Dieu et en partage les propriétés

I8. Voir J. Céard, "'In homine quodam modo sunt omnia'».

19. "Voilà bien pourquoi nous disons que la mère de ce qui est venu à l'être, de ce qui est visible ou du moins perceptible par un sens, c'est-à-dire le réceptacle, n'est ni terre, ni air, ni feu, ni eau, ni rien de tout ce qui vient de ces éléments et de tout ce dont ils dérivent. Mais, si nous disons qu'il s'agit d'une espèce invisible et dépourvue de figure, qui reçoit tout, qui participe de l'intelligible d'une façon particulièrement déconcertante et qui se laisse très difficilement saisir, nous ne mentirions point » (Platon, Timée, p. 149-150 [51a]). 
divines. Le lieu commun du vieillissement du monde - «Le monde est un vieillard dont la folle jeunesse / Lascive, dissolue en toute volupté / Laissa pour apanage à sa faible vieillesse, / Mille malheurs témoins de son infirmité" (p. 39) - prépare bien évidemment la renovatio de l'univers, de même qu'il permet de contrer les ennemis de la foi, "épicuristes" et autres "athées", que Guillaume de Chevalier condamne de la manière la plus véhémente (p. 5).

Cette charpente théologique, en tous points conforme à la doxa, asujettit l'évocation des maux présents qui accablent l'homme à une théodicée. L’œuvre de Chevalier réfléchit le mal, au sens optique du terme, et le fait converger vers un foyer spirituel unique. L'épopée apocalyptique se présente de fait comme le négatif tragique des paraphrases poétiques de la Genèse, dont La Sepmaine ou Creation du monde (1578) de $\mathrm{Du}$ Bartas constitue à n'en pas douter l'archétype. Les merveilles de la Création, signes incontestables de la bonté de Dieu ${ }^{20}$, se voient alors inscrites dans une écriture de l'inversion et de l'abjection. Là où les paraphrases de la Genèse exaltent le devenir grandiose de l'humanité, la poésie apocalyptique conduit l'univers à son néant, peignant ainsi la cohorte de maux - guerre, famine, peste - qui s'abattront successivement sur une humanité corrompue ${ }^{21}$. Ainsi, dans le troisième livre du Decez, ou fin du monde (p. 38-40), Guillaume de Chevalier se sert d'un autre lieu commun, celui du "comble de maux" ou "comble de péchés" qui s'est abattu d'après le récit de l'Apocalypse (XVI, 2) sur l'humanité toute entière; le poète rapproche la corruption des hommes avant la Déluge et les maux présents:

Le temps présent ressemble aux jeunes ans du Monde:

Quand au fils de Lamech le Dieu de l'Univers

Commenda de son bois tout premier fendre l'onde

Dont les corps de ce rond seront alors couverts (p. 37b).

20. La Sepmaine ou Creation du monde (1578) du protestant Du Bartas, l'un des plus grands succès de la seconde moitié du XVI ${ }^{\mathrm{e}}$ siècle, constitue un intertexte privilégié. Même s'il ne cite pas Du Bartas, Guilaume de Chevalier s'en inspire indiscutablement (par son titre, Le Decez, ou fin du monde forme l'exacte inversion de celui de Du Bartas); il en est de même de La Dernière semaine ou Consommation du monde de Michel Quillian.

2I. «Le fer, la faim, la peste ont le bas édifice / Déjà longtemps travaillé par leurs cruelles mains: / Le prodige à sept voix a mis en sacrifice / Les efleuz de l'Esprit qui régit les humains» (p. 5). 
Ce topos, largement exploité dans la littérature apologétique des $\mathrm{XVI}^{\mathrm{e}}$ et XVII siècles - aussi bien du côté catholique que réformé ${ }^{22}-$ fait du temps humain le préalable indispensable à l'accomplissement du règne de Dieu. L'univers a ainsi atteint son apogée négative, laissant au mal (provisoirement du moins) tout pouvoir; la disparition de la vertu, au sens augustinien, entraîne une privation, celle de la forme même. La corruption des hommes se propage dans l'univers et en perturbe l'unité; la nature, elle-même altérée, entrave à son tour le cours de l'histoire humaine. Le Decez, ou fin du monde transpose poétiquement le concept de la regio dissimulitudinis, "cette région où rien ne te ressemble" d'après saint Augustin (Confessions, VII, 10, 16), dont le substrat remonte luimême au Politique de Platon:

Lorsqu'on descend en dessous du vice [...], on se trouve alors dans la région de dissemblance, et, en se plongeant en elle, on s'apprête à tomber dans un bourbier obscur ${ }^{23}$.

La dissemblance est l'avers de la ressemblance avec le divin; c'est une région de mort (l'homme n'est plus temporairement l'imago Dei) dans laquelle l'individu a sombré et de laquelle il ne peut s'extraire par ses propres forces; une région de mort que Guillaume de Chevalier traduit stylistiquement par le recours à l'hyperbole et à une forme de réalisme macabre:

L'air alarme vos camps, les rochers, les montagnes

Du sommeil vicieux veulent ôter votre œil,

Les souffreuses vapeurs qui brulent les campagnes

Veulent tirer vos cœurs de l'infecté cercueil

Les prodiges hideux dont couverte est la Terre

Les forfaits non-ouïs engendrés des horreurs

Au Tartare plus creux du frère du Tonnerre

Ne peuvent arracher vos maudites erreurs (p. 38b).

Seul le temps divin sera à même de dissoudre l'«infirmité» (p. 13) et l'ignorance qui gangrènent l'esprit humain; seul le temps divin permettra cette résurgence du même qui conduira aux fins dernières:

22. Voir E. Forsyth, «D’Aubigné, Calvin et le comble des péchés».

23. Platon, Le Politique, $273 \mathrm{~d}$. 
Toujours le temps s'écoule et sa ferme inconstance

Paraît perpétuelle en ses tours renaissants,

Portrait du grand esprit dont l'infinie essence

Voit les flots les passés, les futurs les présents (p. 26a).

L'évocation du temps est envisagée ici comme la miniature, comme le "portrait» du Créateur; ce faisant, le poème renvoie également à un substrat platonicien qu'il réforme dans le sens de la foi: on se souvient en effet que dans le Timée (38b), le temps est né avec l'univers, dont il est l'image mobile.

Mais le temps de la maladie, après les années de jeunesse, est un temps de préparation à la mort, puis à la renovatio spirituelle et par extension politique. En effet, les maux présents, aussi insupportables qu'ils soient, précèdent néanmoins l'avènement d'une justice véritable, celle qui bannira les vaines hiérarchies mondaines, faisant ainsi taire les divergences confessionnelles qui déchirent le Royaume de France. Le Decez, ou fin $d u$ monde privilégie de toute évidence une remontée vers les vérités divines, propres à faire taire les voix discordantes des hommes; il en est ainsi de l'appel au Roi dans la tradition de la bonne gouvernance (p. 8). En effet, si Guillaume de Chevalier déplore à de nombreuses reprises les exactions commises en son temps (le mal est bien plus grand qu'au temps du déluge biblique, "exécuteur de la vengeance divine», "Epître», p. ii), il se garde pourtant de verser - même indirectement - dans la polémique antiprotestante, privilégiant davantage une forme d'abstraction. Dès lors, les allusions à un "siècle de fer", dans lequel les hommes pétris de péché - c'est le motif de la concupiscence («Epître», p. iii) - s'entretuent, ont une fonction illustrative, mais non exclusive, des maux à venir : ce sont autant de signes qui témoignent de l'imminence de la destruction du monde. Le poète-narrateur devient ainsi un spectateur socratique de la folie des hommes; il témoigne, au sens juridique du terme, de l'alliance - bien évidemment jamais remise en question - entre le Créateur et la créature. Le péritexte du Decez, ou fin du monde énonce d'emblée l'étroite imbrication entre nature et surnature, entre finitude humaine cet éternité divine, entre obscurité et révélation:

L'homme m'a toujours semblé avoir été fait pour imiter l'infinie perfection de son auteur en tant qu'un infini, incorruptible et hors du temps peut être imité d'un fini, corruptible et sujet à l'empire du temps 
qui n'est autre chose que le mouvement de chaque être sensible qui ne demeure jamais en même état ainsi, comme un Protée emprunte une infinité de divers masques jouant divers personnages en un même sujet inquiet («Epître», p. i).

Véritable "Protée " ${ }^{24}$, l'être humain est entièrement guidé par une pulsion métamorphique (l'adjectif «inquiet» renvoie ici à sa signification étymologique première: in-quies). Soumis à la corruption - entendue ici dans un sens augustinien ${ }^{25}$ - le sujet est une figure composite, traversée par des éléments conflictuels, en apparence inconciliables. L'insuffisance de la raison humaine, autre lieu commun de l'apologétique catholique et réformée, nourri en outre par l'émergence du scepticisme au cours de la seconde moitié du XVI $\mathrm{I}^{\mathrm{e}}$ siècle, conduit certes à une leçon d'humilité. L'apport théologique d'une telle conception des représentations mondaines se conçoit aisément: même marquée ontologiquement par le péché, la nature humaine n'est pas pour autant irrémissible; en dépit de sa difformitas, l'individu n'a pas perdu pour autant sa vocation originelle, bien qu'il n'en possède plus les attributs; il sera rétabli par l'Incarnation dans la vérité et dans la beauté, et recouvrera son origine divine ${ }^{26}$. En tant que réduction imparfaite du divin, tout homme doit tendre vers la perfection, incarnée par le hors-temps divin. Il est à souligner que les enjeux sont ici autant doctrinaux que confessionnels: croire à la seule prédestination comme le font les réformés, c'est invalider le lien prototypique qui unit l'homme et son Créateur ${ }^{27}$. S'il y a une prédestination, elle ne peut être qu'une prédestination au salut, étant donné qu'elle nécessite pleinement l'adhésion humaine; le salut par la grâce, sans le

24. Le terme apparaît également dans le poème: «Les choses du bas monde ont un être passible, / Un Protée inconstant qui reçoit les portraits / Qui des fleuves naissants de l'onde corruptible / Sans cesse tournoyant du changement sont faits" (p. 25).

25. Pour l'évêque d'Hippone, les êtres créés se caractérisent par une défaillance ontologique qui touche au néant lorsque Dieu se retire du monde, renonçant alors à sa fonction fabricatrice: «Il y a donc un bien inaltérable: Dieu; et tous les autres, qui viennent de lui, sont en eux-mêmes susceptibles d'altération, parce que d'eux-mêmes, ils ne sont rien" (De Vera religione, XIX, 37).

26. Sur le couple difformitas-reformitas, voir C. Harrison, Beauty and Revelation in the Thought of Saint Augustine.

27. La qualification de l'homme en tant qu'imago ad imaginem apparaît dès l'«Epître» (p. i), marque indiscutable de l'orientation théologique du poème. 
concours du libre-arbitre, conduirait à placer Dieu hors d'atteinte et à exacerber la notion de péché.

Fondée sur le principe de l'amplification, la matière apocalyptique telle que la présente Le Decez, ou fin du monde de Chevalier se lit bien entendu à la lumière de la Chute et de la rédemption: la corruption du monde terrestre, de même que les faiblesses humaines, ne constituent aucunement un obstacle au règne de Dieu, mais bien au contraire la condition indispensable à sa réalisation. Il y a dans la figuration poétique des fins dernières et de la corruption qui anime le monde terrestre un spectacle du sens, c'est-à-dire le dévoilement d'un mystère transcendant, qui revêt en retour une fonction probatoire. En cela, l'œuvre se donne à lire comme une épiphanie, c'est-à-dire comme le surgissement d'un monde à venir dont il s'agit d'expliquer les desseins divins qui y président; l'imagination, guidée par la puissance de l'éloquence sacrée, conduit à un croire. La confrontation de la corruptibilité sublunaire et l'incorruptibilité divine témoigne plus largement de la volonté du poète de réactualiser le récit biblique dans le présent vécu du narrateur et $\mathrm{du}$ lecteur. Cette dimension est rendue lexicalement par les très nombreuses occurrences du verbe "voir", énoncé au présent. Le dispositif verbal permet d'asseoir la convergence du fini et de l'infini et a pour effet d'accroître la participation sensible, quasi cénesthésique du lecteur; le poète-narrateur apparaît de son côté comme un témoin, un chroniqueur des fins dernières. La restitution poétique de l'Apocalypse s'attèle ainsi à combler un écart par essence impossible à dépasser qui atteste a posteriori du sublime de l'épopée biblique et, par là même, de l'ethos du poète. C'est à dessein que Guillaume de Chevalier use (et abuse parfois) d'un vaste réseau d'antithèses; cette solidarité des contraires a pour effet de défier la raison du lecteur et de décentrer son regard, troublant ainsi les repères cognitifs qui sont les siens. C'est une matière poétique qui croit en la convergence du signe et du référent, même si ce dernier se situe «hors du temps» ("Epître», p. i); elle ne saurait toutefois se situer sur le plan d'une forme d'alchimie poétique qui se contenterait de manier des réseaux de correspondances; de ce point de vue, le Decez, ou fin $d u$ monde s'apparie de plain-pied à une célébration poétique du déchiffrement du monde. C'est pour cela que le poème effectue un parcours circulaire, accompagné de nombreuses digressions (Guillaume de Chevalier se fait ici l'émule de Du Bartas), mêlant en outre plusieurs champs de 
savoir, afin de restituer les mystères divins, le tout se définissant comme une vision ordonnée et cohérente du monde.

Adrien Paschoud

Université de Bâle 


\section{BIBLIOGRAPHIE}

\section{Textes}

Calvin, Jean, Contre les libertins [1544], in Euvres, éd. par Francis Higman, Bernard Roussel, Paris, Gallimard, 2009 (Bibliothèque de la Pléiade).

Chevalier, Guillaume de, Le Decez, ou fin du Monde, divisé en trois visions, Paris, R. Le Fizelier ou P. Sevestre, 1584.

Quillian, Michel, La Dernière semaine ou Consommation du monde, A Rouen, C. Le Villain, 1597.

Platon, Timée. Critias, éd. par Luc Brisson, Paris, GF, 2001.

\section{Travaux}

Charpentier, Françoise (éd.), Le songe à la Renaissance, Saint-Etienne, Presses de l'Université de Saint-Etienne, 1990.

CÉARD, Jean, "'In homine quodam modo sunt omnia': Récapitulation et analogie à la Renaissance", in Macrocosmo-microcosmo. Scrivere e pensare il mondo nel Cinquecento tra Italia e Francia. Atti del Convegno Internazionale di Studio, Verona, 23-25 maggio 2002, a cura di Rosanna Gorris Camos, Fasano, Schena Editore, 2004, p. 11-22.

Demonet, Marie-Luce, La voix du signe. Nature et origine du langage à la Renaissance (1480-1580), Paris, Honoré Champion, 1992.

Forsyth, Elliott, "D’Aubigné, Calvin et le comble des péchés", in Mélanges sur la littérature de la Renaissance à la mémoire de V.-L. Saulnier, éd. par Pierre-Georges Castex, Genève, Droz, 1984, p. 263-272.

Harrison, Carol, Beauty and Revelation in the Thought of Saint Augustine, Oxford, Oxford University Press, 1992. 
Lecompte, Stéphanie, La chaîne d'or des poètes. Présence de Macrobe dans l'Europe humaniste, Genève Droz, 2009.

MÉniel, Bruno, Renaissance de l'épopée. La poésie épique en France de 1572 à 1623, Genève, Droz, 2004.

Miernowski, Jan, Signes dissimilaires: la quête des noms divins dans la poésie française de la Renaissance, Genève, Droz, 1997.

Orwat, Florence, L'invention de la rêverie. Une conquête pacifique du Grand Siècle, Paris, Honoré Champion, 2006.

Pantin, Isabelle, La poésie du ciel en France dans la seconde moitié du seizième siècle, Genève, Droz, 1995.

Spica, Anne-Elisabeth, Symbolique humaniste et emblématique: l'évolution et les genres (1580-1700), Paris, Honoré Champion, 1996.

Victoria, Thierry, Un livre de feu dans un siècle de fer. Les lectures de l'Apocalypse dans la littérature française de la Renaissance, Louvain, Peeters, 2009. 